\title{
ON THE UBIQUITY OF MOLECULAR ANIONS IN THE DENSE INTERSTELLAR MEDIUM
}

\author{
M. A. Cordiner ${ }^{1,2}$, J. V. BuCKLE ${ }^{3}$, E. S. Wirström ${ }^{1,4}$, A. O. H. OlofsSON ${ }^{4}$, And S. B. Charnley ${ }^{1}$ \\ ${ }^{1}$ Astrochemistry Laboratory and The Goddard Center for Astrobiology, NASA Goddard Space Flight Center, Code 691, \\ 8800 Greenbelt Road, Greenbelt, MD 20771, USA; martin.cordiner@ nasa.gov \\ ${ }^{2}$ Department of Physics, The Catholic University of America, Washington, DC 20064, USA \\ ${ }^{3}$ Cavendish Astrophysics Group and Kavli Institute for Cosmology, Institute of Astronomy, University of Cambridge, Madingley Road, Cambridge CB3 0HE, UK \\ ${ }^{4}$ Department of Earth and Space Sciences, Chalmers University of Technology, Onsala Space Observatory, SE-439 92 Onsala, Sweden \\ Received 2012 December 13; accepted 2013 April 1; published 2013 May 23
}

\begin{abstract}
Results are presented from a survey for molecular anions in seven nearby Galactic star-forming cores and molecular clouds. The hydrocarbon anion $\mathrm{C}_{6} \mathrm{H}^{-}$is detected in all seven target sources, including four sources where no anions have been previously detected: $\mathrm{L} 1172, \mathrm{~L} 1389, \mathrm{~L} 1495 \mathrm{~B}$, and TMC-1C. The $\mathrm{C}_{6} \mathrm{H}^{-} / \mathrm{C}_{6} \mathrm{H}$ column density ratio is $\gtrsim 1.0 \%$ in every source, with a mean value of $3.0 \%$ (and standard deviation $0.92 \%$ ). Combined with previous detections, our results show that anions are ubiquitous in dense clouds wherever $\mathrm{C}_{6} \mathrm{H}$ is present. The $\mathrm{C}_{6} \mathrm{H}^{-} / \mathrm{C}_{6} \mathrm{H}$ ratio is found to show a positive correlation with molecular hydrogen number density, and with the apparent age of the cloud. We also report the first detection of $\mathrm{C}_{4} \mathrm{H}^{-}$in TMC-1 (at $4.8 \sigma$ confidence), and derive an anion-to-neutral ratio $\mathrm{C}_{4} \mathrm{H}^{-} / \mathrm{C}_{4} \mathrm{H}=(1.2 \pm 0.4) \times 10^{-5}(=0.0012 \% \pm 0.0004 \%)$. Such a low value compared with $\mathrm{C}_{6} \mathrm{H}^{-}$highlights the need for a revised radiative electron attachment rate for $\mathrm{C}_{4} \mathrm{H}$. Chemical model calculations show that the observed $\mathrm{C}_{4} \mathrm{H}^{-}$could be produced as a result of reactions of oxygen atoms with $\mathrm{C}_{5} \mathrm{H}^{-}$and $\mathrm{C}_{6} \mathrm{H}^{-}$.
\end{abstract}

Key words: astrochemistry - ISM: abundances - ISM: clouds - ISM: molecules - stars: formation

Online-only material: color figures

\section{INTRODUCTION}

Negative ions (anions) were first discovered in the interstellar medium (ISM) by McCarthy et al. (2006), who detected the linear hydrocarbon anion $\mathrm{C}_{6} \mathrm{H}^{-}$in TMC-1. This was followed by $\mathrm{C}_{6} \mathrm{H}^{-}$and $\mathrm{C}_{4} \mathrm{H}^{-}$detections in the protostellar core $\mathrm{L} 1527$ by Sakai et al. (2007) and Agúndez et al. (2008), respectively, which hinted of a role for anions in the chemistry of star formation. Gupta et al. (2009) performed the first dedicated survey for $\mathrm{C}_{6} \mathrm{H}^{-}$in 24 molecular sources, but detected the anion in only two star-forming clouds, with anion-to-neutral ratios on the order of a few percent, similar to previously observed values. Interstellar anion detections were confined to the Taurus molecular cloud complex until the recent discoveries of $\mathrm{C}_{6} \mathrm{H}^{-}$in the Lupus, Cepheus, and Auriga star-forming regions (Sakai et al. 2010; Cordiner et al. 2011), which proved that anions are widespread in the local ISM, and not an artifact of any particular physical or chemical conditions in the Taurus region.

The possible importance of anions in interstellar chemistry was first discussed by Dalgarno \& McCray (1973), and prospects for the detection of molecular anions using radio astronomy were examined by Sarre (1980). Herbst (1981) argued that large interstellar molecules (including carbonchain-bearing species) can undergo rapid radiative electron attachment-as exemplified by the laboratory experiments of Woodin et al. (1980) - potentially resulting in significant anion abundances in dense molecular clouds. Based on this idea, models for anion chemistry have been successful in reproducing the observed abundances of $\mathrm{C}_{6} \mathrm{H}^{-}$and $\mathrm{C}_{8} \mathrm{H}^{-}$in TMC-1, IRC+10216, L1527, and L1512 (Millar et al. 2007; Remijan et al. 2007; Harada \& Herbst 2008; Cordiner et al. 2008, 2012). However, there is a major discrepancy between the modeled and observed $\mathrm{C}_{4} \mathrm{H}^{-}$anion-to-neutral ratio (see Herbst \& Osamura 2008), and the lack of anions in photon-dominated regions (Agúndez et al. 2008) is at variance with the model predictions of Millar et al. (2007). Clearly, our understanding of molecular anion chemistry is incomplete. Nevertheless, as demonstrated by Cordiner et al. (2012), anion measurements have the potential to offer insight into interstellar cloud properties due to their reactivity (Eichelberger et al. 2007), and consequently, their sensitivity to the abundances of gas-phase electrons and atomic C, $\mathrm{H}$, and $\mathrm{O}$, and to physical conditions such as cloud density and molecular depletion. Observations of anions in a variety of interstellar environments will be key to a complete understanding of their chemistry. Given the relatively small number of molecular anion detections in the ISM to date, we set out to address the question of just how widespread anions are in interstellar clouds, and to ascertain the behavior of the anion-to-neutral ratio over a range of cloud types and ages.

In this article, results are presented from our survey for $\mathrm{C}_{6} \mathrm{H}^{-}$ and $\mathrm{C}_{6} \mathrm{H}$ in a sample of seven carbon-chain-rich interstellar clouds, protostars, and prestellar cores. Detections of $\mathrm{C}_{6} \mathrm{H}^{-}$ in two of the sources (L1251A and L1512) were previously reported by Cordiner et al. (2011), and here we report the final results for all seven sources, as well as the first (tentative) detection of $\mathrm{C}_{4} \mathrm{H}^{-}$in TMC-1.

\section{TARGET SOURCES}

Due to the close chemical relationship between polyynes and cyanopolyynes (see, e.g., Federman et al. 1990; Millar \& Herbst 1994), carbon-chain-bearing species such as $\mathrm{C}_{4} \mathrm{H}$ and $\mathrm{C}_{6} \mathrm{H}$ are expected to be most abundant in dense molecular clouds near to the peak of $\mathrm{HC}_{3} \mathrm{~N}$ emission. Target sources with strong $\mathrm{HC}_{3} \mathrm{~N}$ emission lines were selected from an $\mathrm{HC}_{3} \mathrm{~N} J=10-9$ mapping survey of 20 nearby molecular clouds, prestellar cores, and young, low-mass protostars obtained using the Onsala Space Observatory (OSO) $20 \mathrm{~m}$ telescope between 2005 and 2012. Some of these data were presented by Buckle et al. (2006) and Cordiner et al. (2011). Maps of the integrated $\mathrm{HC}_{3} \mathrm{~N} J=10-9$ emission intensity toward six of our seven chosen sources (L1172, L1251A, L1389, L1495B, L1512, and TMC-1C) are 
Table 1

Targets, Coordinates, and Distances

\begin{tabular}{lcclcc}
\hline \hline Source & $\begin{array}{c}\text { R.A. } \\
(\text { J2000 })\end{array}$ & $\begin{array}{c}\text { Decl. } \\
(\text { J2000) }\end{array}$ & Cloud Type $^{\text {a }}$ & $\begin{array}{c}\text { Distance } \\
(\mathrm{pc})\end{array}$ & Ref. \\
\hline L1172 SMM & $21: 02: 22.1$ & $+67: 54: 48$ & Star forming & 440 & 1 \\
L1251A & $22: 30: 40.4$ & $+75: 13: 46$ & Star forming & 300 & 2 \\
L1389 (CB17) SMM1 & $04: 04: 36.6$ & $+56: 56: 00$ & Protostellar & 250 & 3 \\
L1495B & $04: 15: 41.8$ & $+28: 47: 46$ & Quiescent & 140 & 4 \\
L1512 & $05: 04: 07.1$ & $+32: 43: 09$ & Star forming & 140 & 4 \\
TMC-1C & $04: 41: 35.6$ & $+26: 00: 21$ & Star forming & 140 & 4 \\
TMC-1 CP & $04: 41: 41.9$ & $+25: 41: 27$ & Quiescent & 140 & 4 \\
\hline
\end{tabular}

Note.

a See Section 2 for description of source classification scheme.

References. (1) Visser et al. 2002; (2) Kun \& Prusti 1993; (3) Launhardt et al. 2010; (4) Elias 1978.

shown in Figures 1 and 2. These observations were obtained using the OSO with a beam size of $42^{\prime \prime}$ and beam efficiency of $0.5 \pm 0.05$. A $30^{\prime \prime}$ map spacing was used, which was resampled to a $7^{\prime \prime} .5$ pixel $^{-1}$ grid using bilinear interpolation. For TMC-1, $\mathrm{HC}_{3} \mathrm{~N}$ maps were published by Hirahara et al. (1992) and Pratap et al. (1997).

To search for $\mathrm{C}_{6} \mathrm{H}$ and $\mathrm{C}_{6} \mathrm{H}^{-}$, we used the $100 \mathrm{~m}$ Robert $\mathrm{C}$. Byrd Green Bank Telescope (GBT) and targeted the strongest $\mathrm{HC}_{3} \mathrm{~N}$ peak positions in our maps of L1389, L1495B, L1512, and TMC-1C. For L1172 we targeted the submillimeter core cataloged by Di Francesco et al. (2008), which fell within $15^{\prime \prime}$ of the $\mathrm{HC}_{3} \mathrm{~N}$ peak. Our chosen L1251A position does not coincide with the main $\mathrm{HC}_{3} \mathrm{~N}$ peak because it was based on an earlier, lower-sensitivity map than that shown in Figure 1. For TMC-1, we targeted the cyanopolyyne peak (CP) where Agúndez et al. (2008) previously attempted to detect $\mathrm{C}_{4} \mathrm{H}^{-}$. The adopted coordinates for our anion survey targets are listed in Table 1.

Our L1172 and L1512 $\mathrm{C}_{6} \mathrm{H}^{-}$positions overlap the prestellar cores identified by Visser et al. (2002) and Ward-Thompson et al. (1994), respectively. For L1389, the $\mathrm{HC}_{3} \mathrm{~N}$ emission maps out a very compact clump, coincident with the center of the Bok Globule CB17 (Clemens \& Barvainis 1988). The $\mathrm{HC}_{3} \mathrm{~N}$ peak also matches closely the location of strongest microwave/submillimeter continuum emission observed by Launhardt et al. (2010) and denoted L1389 SMM1 (the position of which is indicated in the bottom panel of Figure 1 by the cross inside the dashed black circle of the GBT beam). As shown in Figure 1, our targeted L1251A position is $\approx 40^{\prime \prime}$ southeast of the center of the protostar L1251 IRS3 (Lee et al. 2010), although the outer envelope of this protostar probably intersects the GBT beam to some extent, as discussed by Cordiner et al. (2011).

From dust continuum emission, Launhardt et al. (2010) obtained a number density for L1389 SMM1 of $6 \times 10^{6} \mathrm{~cm}^{-3}$. Pavlyuchenkov et al. (2006) derived a moderately high CO depletion factor $(\sim 40)$, and inferred a relatively evolved chemical age of $\sim 2$ Myr for this collapsing core. The core was recently found to contain a very young, low-luminosity protostar (referred to as CB17 MMS; Chen et al. 2012), 8" NE of our targeted GBT beam center, as well as a more-evolved Class 0/I protostar $20^{\prime \prime} \mathrm{NW}$. Given that the radii of typical low-mass protostellar envelopes are $\sim 10^{4}$ AU (e.g., Jørgensen et al. 2002), at a distance of $250 \mathrm{pc}$, our targeted L1389 beam is likely to be dominated by protostellar envelope matter.

L1495B, on the other hand, is a quiescent molecular cloud with no known protostars nearby. It appears to be chemically
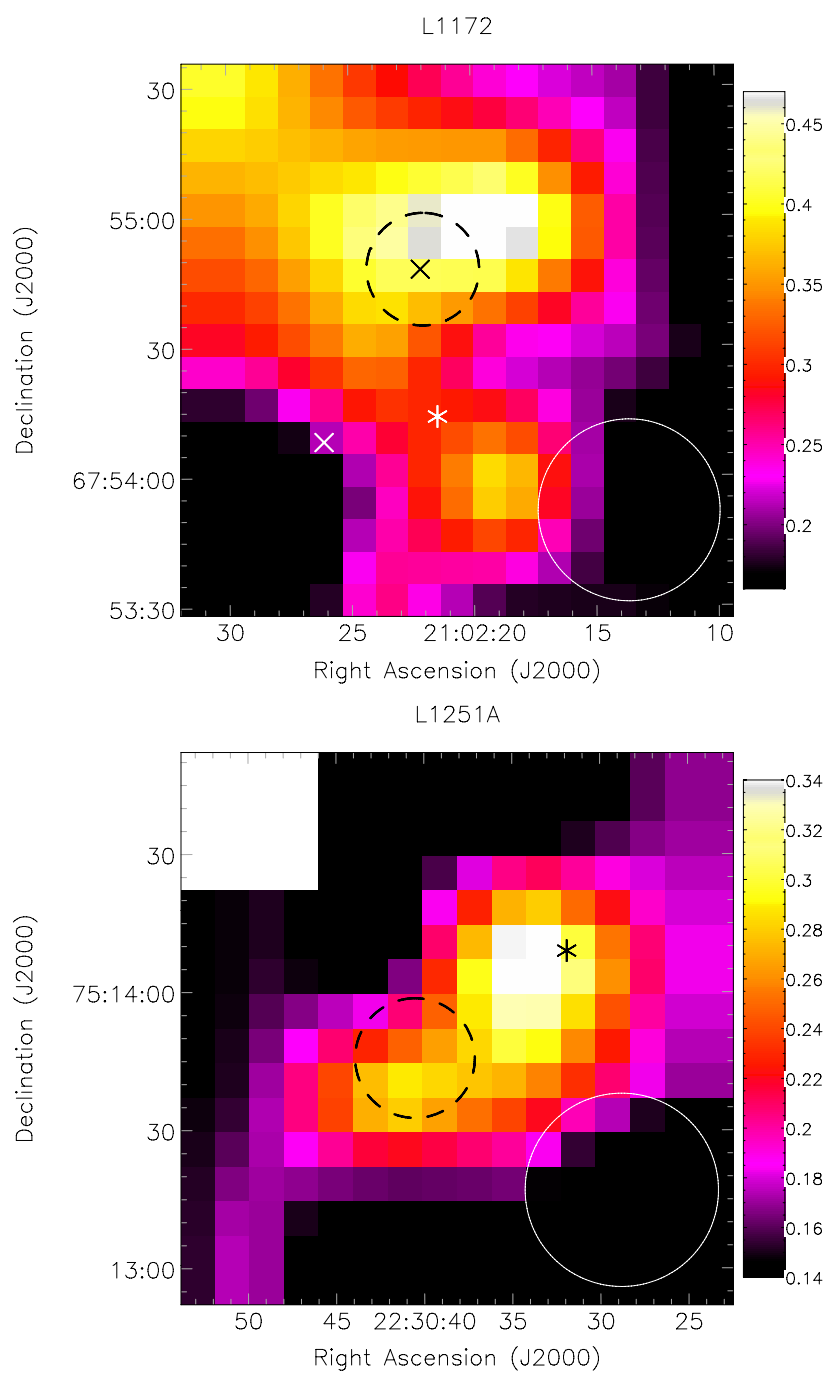

L1389

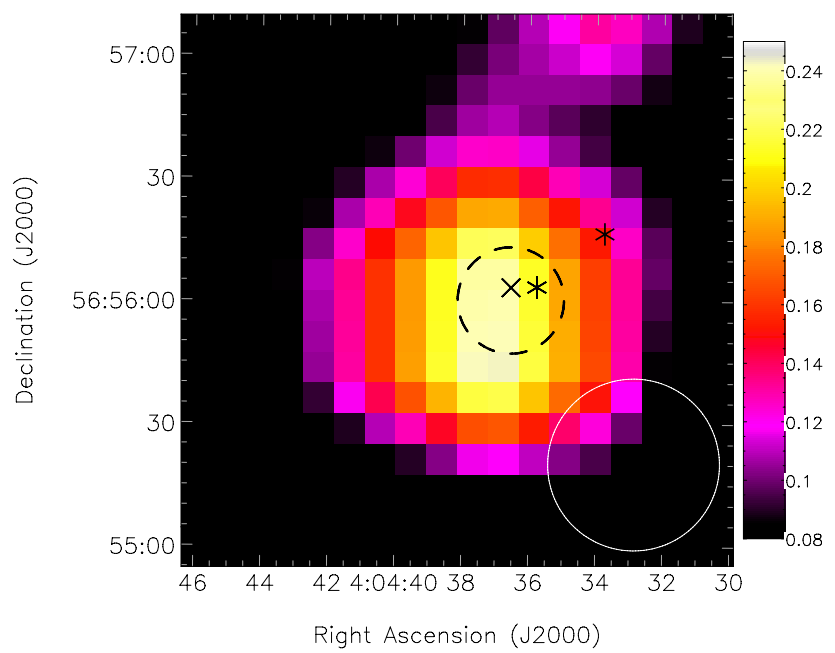

Figure 1. Observed $\mathrm{HC}_{3} \mathrm{~N} J=10-9$ integrated intensity maps toward $\mathrm{L} 1172$, L1251A, and L1389. The OSO beam size is indicated in the lower right of each panel with a white circle. Intensity scales are given in units of $\mathrm{K} \mathrm{km} \mathrm{s}^{-1}$ and have not been corrected for beam efficiency. Dashed black circles represent the size (HPBW) and position of our targeted GBT anion survey positions. Crosses denote locations of submillimeter (prestellar) cores (Ward-Thompson et al. 1994; Visser et al. 2002; Di Francesco et al. 2008; Launhardt et al. 2010) and asterisks denote protostellar core locations (Visser et al. 2002; Chen et al. 2012; Lee et al. 2010).

(A color version of this figure is available in the online journal.) 

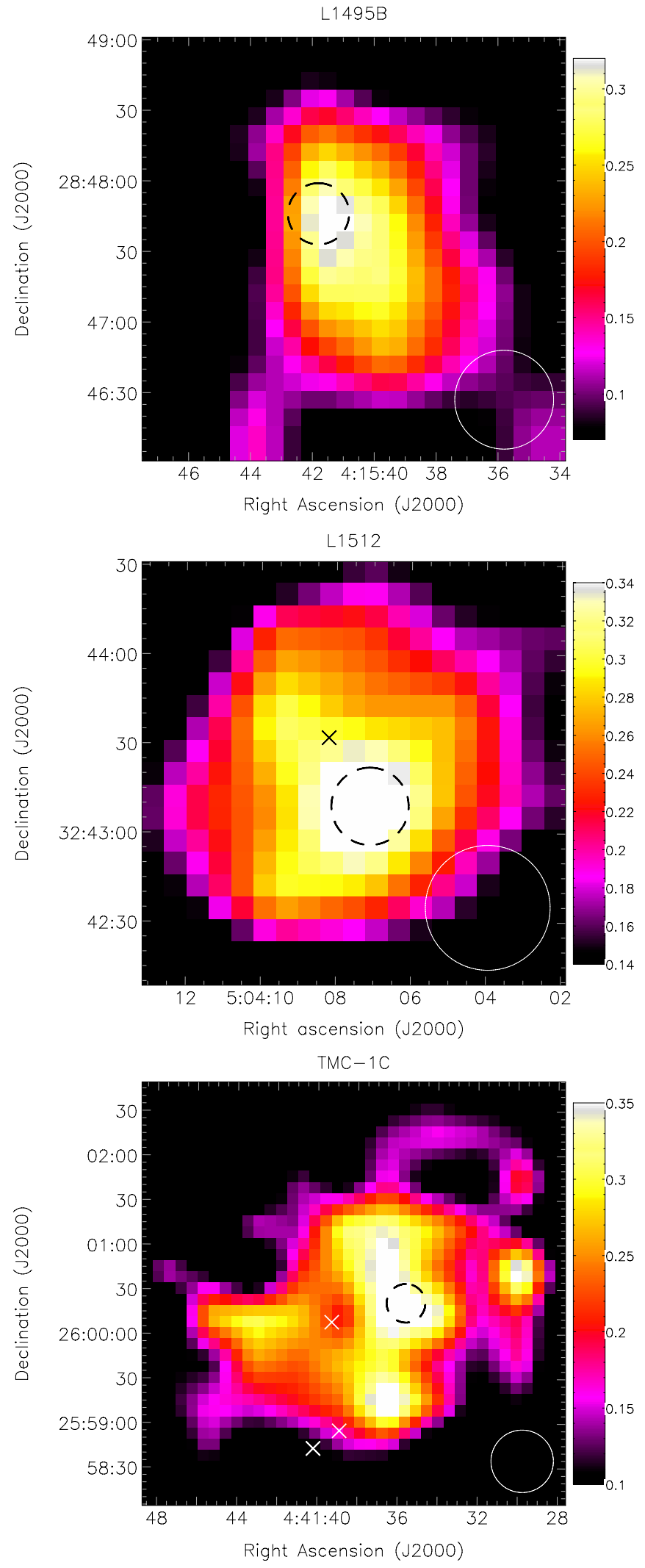

Figure 2. Same as Figure 1, for L1495B, L1512, and TMC-1C. (A color version of this figure is available in the online journal.)

young, with an apparently low level of $\mathrm{CO}$ depletion consistent with less-evolved interstellar clouds (Hirota et al. 2004). In TMC-1C, Buckle et al. (2006) identified an anti-correlation between the spatial distributions of $\mathrm{HC}_{3} \mathrm{~N}$ and $\mathrm{C}^{18} \mathrm{O}$, at least partly attributable to $\mathrm{CO}$ depletion. Thus, our observations of TMC-1C (at the $\mathrm{HC}_{3} \mathrm{~N}$ peak) probably sample more chemically evolved, depleted gas than L1495B. No protostars are known to be present in our observed TMC-1C beam, but several submillimeter sources are located in the surrounding cloud (indicated by white crosses in the bottom panel of Figure 2), showing this to be a region of active star formation. Southwest of TMC-1C lies the source TMC-1 (CP), which is a well-known chemically young dark cloud, with only a modest degree of depletion (Hirahara et al. 1992; Cordiner et al. 2012).

In Table 1, we provide a basic categorization of our target sources in light of their properties described above: "quiescent" refers to those cloud cores that are chemically young, show no evidence for active star formation within the telescope beam (such as outflows or compact submillimeter/IR emission), and do not appear to be undergoing collapse; "star forming" is used for clouds showing nearby active star formation and a greater degree of chemical evolution; "protostellar" describes our L1389 position, which contains the low-luminosity protostar CB17 MMS.

\section{ANION OBSERVATIONS}

Observations of emission lines of $\mathrm{C}_{6} \mathrm{H}(J=10.5-9.5), \mathrm{C}_{6} \mathrm{H}^{-}$ ( $J=10-9$ and 11-10), and $\mathrm{HC}_{3} \mathrm{~N}(J=3-2$ and $4-3)$ were carried out between 2010 April and 2011 January using the Ka receiver of the GBT. The GBT Spectrometer was used with a bandwidth of $50 \mathrm{MHz}$ and $8192 \times 6.1 \mathrm{kHz}$ channels (corresponding to a velocity spacing of $\approx 0.065 \mathrm{~km} \mathrm{~s}^{-1}$ ). Four spectral windows were used, which allowed simultaneous observation of the $\mathrm{C}_{6} \mathrm{H}$ and $\mathrm{C}_{6} \mathrm{H}^{-}$lines. For the compact, spatially isolated source L1512, beam switching (with 78" throw) was used, and for all other targets, frequency switching was used. Pointing was checked every one to two hours and was typically accurate to within $5^{\prime \prime}$. In the middle of the observed frequency range $(28 \mathrm{GHz})$, the telescope beam FWHM was $26^{\prime \prime}$ and the main beam efficiency was 0.90 . Total system temperatures were typically in the range $60-80 \mathrm{~K}$ and the zenith opacity was $0.05 \pm 0.02$. Intensity calibration was performed using beamswitched observations of the compact radio source NGC 7027. Measured antenna temperatures were subsequently corrected for opacity, spillover, ohmic loss, blockage efficiency, and beam efficiency, then averaged using standard GBTIDL routines.

We obtained observations of $\mathrm{C}_{4} \mathrm{H}(N=2-1, J=1.5-0.5$, $F=2-1)$ and $\mathrm{C}_{4} \mathrm{H}^{-}(J=2-1)$ between 2011 December and 2012 February at around $19 \mathrm{GHz}$ using the GBT $K$-band focalplane array. These observations were obtained with channel spacing $12.2 \mathrm{kHz}$, beam efficiency 0.92 , beam size $39^{\prime \prime}$, and zenith opacity $0.025 \pm 0.015$.

Additional single-pointing $\mathrm{HC}_{3} \mathrm{~N} J=10-9$ spectra of our sources were obtained using the OSO at our adopted target positions. A list of all the observed transitions is given in Table 2.

\section{RESULTS}

\subsection{New Anion Detections}

The $\mathrm{C}_{6} \mathrm{H}^{-}$anion and its neutral counterpart $\mathrm{C}_{6} \mathrm{H}$ were detected in all of the surveyed sources. This is the first time anions have been detected in L1172, L1389, L1495B, and TMC-1C. The observed $\mathrm{C}_{6} \mathrm{H}^{-}$and $\mathrm{C}_{6} \mathrm{H}$ spectra are shown in Figure 3.

Spectra of the $J=10-9$ and $J=11-10$ transitions of $\mathrm{C}_{6} \mathrm{H}^{-}$ were averaged in velocity space to improve the signal-to-noise 
Table 2

Observed Species, Transitions, and Frequencies

\begin{tabular}{lccccc}
\hline \hline Species & Transition & $\begin{array}{c}\text { Frequency } \\
(\mathrm{MHz})\end{array}$ & Ref. & Telescope & $\begin{array}{c}\text { HPBW } \\
\left({ }^{\prime}\right)\end{array}$ \\
\hline $\mathrm{C}_{4} \mathrm{H}$ & $N=2-1, J=1.5-0.5, F=2-1$ & 19054.4760 & 1 & GBT & 38 \\
$\mathrm{C}_{4} \mathrm{H}^{-}$ & $J=2-1$ & 18619.7580 & 2,3 & GBT & 39 \\
$\mathrm{C}_{6} \mathrm{H}^{-}$ & $J=10-9$ & 27537.1302 & 4 & GBT & 26 \\
$\mathrm{C}_{6} \mathrm{H}^{-}$ & $J=11-10$ & 30290.8133 & 4 & GBT & 24 \\
$\mathrm{C}_{6} \mathrm{H}$ & $J=10.5-9.5, f, F=11-10$ & 29109.6437 & 5 & GBT & 25 \\
$\mathrm{C}_{6} \mathrm{H}$ & $J=10.5-9.5, f, F=10-9$ & 29109.6855 & 5 & GBT & 25 \\
$\mathrm{C}_{6} \mathrm{H}$ & $J=10.5-9.5, e, F=11-10$ & 29112.7087 & 5 & GBT & 25 \\
$\mathrm{C}_{6} \mathrm{H}$ & $J=10.5-9.5, e, F=10-9$ & 29112.7503 & 5 & GBT & 25 \\
$\mathrm{HC}_{3} \mathrm{~N}$ & $J=3-2, F=3-3$ & 27292.9018 & 6 & GBT & 27 \\
$\mathrm{HC}_{3} \mathrm{~N}$ & $J=3-2, F=2-1$ & 27294.0758 & 6 & GBT & 27 \\
$\mathrm{HC}_{3} \mathrm{~N}$ & $J=3-2, F=3-2$ & 27294.2927 & 6 & GBT & 27 \\
$\mathrm{HC}_{3} \mathrm{~N}$ & $J=3-2, F=4-3$ & 27294.3451 & 6 & GBT & 27 \\
$\mathrm{HC}_{3} \mathrm{~N}$ & $J=3-2, F=2-2$ & 27296.2334 & 6 & GBT & 27 \\
$\mathrm{HC}_{3} \mathrm{~N}$ & $J=4-3, F=4-4$ & 36390.8861 & 6 & GBT & 20 \\
$\mathrm{HC}_{3} \mathrm{~N}$ & $J=4-3, F=3-2$ & 36392.2358 & 6 & GBT & 20 \\
$\mathrm{HC}_{3} \mathrm{~N}$ & $J=4-3, F=4-3$ & 36392.3293 & 6 & GBT & 20 \\
$\mathrm{HC}_{3} \mathrm{~N}$ & $J=4-3, F=5-4$ & 36392.3630 & 6 & GBT & 20 \\
$\mathrm{HC}_{3} \mathrm{~N}$ & $J=4-3, F=3-3$ & 36394.1765 & 6 & GBT & 20 \\
$\mathrm{HC}_{3} \mathrm{~N}$ & $J=10-9, F=9-8$ & 90978.9838 & 6 & OSO & 42 \\
$\mathrm{HC}_{3} \mathrm{~N}$ & $J=10-9, F=10-9$ & 90978.9948 & 6 & OSO & 42 \\
$\mathrm{HC}_{3} \mathrm{~N}$ & $J=10-9, F=11-10$ & 90979.0024 & 6 & OSO & 42 \\
& & & & &
\end{tabular}

Note.

a Telescope half-power beam width at observed frequency.

References. (1) Gottlieb et al. 1983; (2) Gupta et al. 2007; (3) McCarthy \& Thaddeus 2008; (4) McCarthy et al. 2006; (5) McCarthy \& Thaddeus 2005; (6) $\mathrm{HC}_{3} \mathrm{~N}$ line predictions are based on spectroscopic data summarized by Thorwirth et al. (2000) and de Zafra (1971).

ratio; integrated intensities of the averaged spectra are given in Table 3. Due to partial blending of the hyperfine components of the $\mathrm{C}_{6} \mathrm{H}(\mathrm{J}=10.5-9.5, f)$ lines, the integrated intensity summed over both components is given in Table 3. Given the weakness of these lines, they can safely be assumed to be optically thin, and Equation (2) of Lis et al. (2002) was used to derive column densities for $\mathrm{C}_{6} \mathrm{H}$ and $\mathrm{C}_{6} \mathrm{H}^{-}$(given in Table 3 ). Spectroscopic data for the transitions of interest (Table 2) were obtained from the Cologne Database for Molecular Astronomy (Müller et al. 2005), and an excitation temperature of $10 \mathrm{~K}$ was assumed. Error estimates were derived using 500 Monte Carlo noise replications for each measurement.

In TMC-1, $\mathrm{C}_{6} \mathrm{H}$ excitation temperatures of $5.2 \mathrm{~K}$ and $6.7 \mathrm{~K}$ were measured by Bell et al. (1999) and Sakai et al. (2007), respectively. Such sub-thermal excitation might be expected given the large Einstein $A$ coefficients for rotational transitions of this molecule, which are a direct consequence of its large (5.5 D) dipole moment. However, the value obtained by Bell et al. (1999) may be subject to uncertainty due to the effects of telescope beam dilution, and the Sakai et al. (2007) value may not be applicable to our observations because it was obtained using the $J=16.5-15.5$ transition, which has an Einstein $A$ coefficient about four times greater than that of the $J=10.5-9.5$ transition we observed. The larger Einstein $A$ results in an increased likelihood of sub-thermal excitation of the $J=16.5$ level. If the $\mathrm{C}_{6} \mathrm{H}$ excitation temperature is as low as $5 \mathrm{~K}$, then the calculated column densities for $\mathrm{C}_{6} \mathrm{H}$ and $\mathrm{C}_{6} \mathrm{H}^{-}$will be about $36 \%$ smaller than the values in Table 3 . Due to the similar moments of inertia of $\mathrm{C}_{6} \mathrm{H}$ and $\mathrm{C}_{6} \mathrm{H}^{-}$, their rotational levels occur at similar energies, so that the calculated anion-to-neutral column density ratios $\left(\mathrm{C}_{6} \mathrm{H}^{-} / \mathrm{C}_{6} \mathrm{H}\right)$ are insensitive to small uncertainties in excitation, provided both molecules share a common excitation temperature. The mean $\mathrm{C}_{6} \mathrm{H}^{-} / \mathrm{C}_{6} \mathrm{H}$ ratio for the seven sources is $3.0 \%$, with a standard deviation of $0.92 \%$.

Our new measurements for $\mathrm{C}_{6} \mathrm{H}$ and $\mathrm{C}_{6} \mathrm{H}^{-}$in L1251A and L1512 supersede those presented by Cordiner et al. (2011). Although the column densities and anion-to-neutral ratios measured in the present study match those of Cordiner et al. (2011) within the stated errors, the newer values benefit from improved calibration and analysis methods and have smaller uncertainties. The difference between our TMC-1 (CP) $\mathrm{C}_{6} \mathrm{H}^{-} / \mathrm{C}_{6} \mathrm{H}$ value of $2.5 \% \pm 0.4 \%$ and the value of $1.6 \% \pm 0.3 \%$ obtained by Brünken et al. (2007) is just beyond the range of the $1 \sigma$ error bars, and could be the result of statistical noise in the spectra. Alternatively, because Brünken et al. (2007) derived their value from a different set of transitions, non-LTE excitation effects in $\mathrm{C}_{6} \mathrm{H}$ and/or $\mathrm{C}_{6} \mathrm{H}^{-}$could be partially responsible for the discrepancy.

The observed $\mathrm{C}_{4} \mathrm{H}^{-}$and $\mathrm{C}_{4} \mathrm{H}$ spectra of TMC-1 (CP) are shown in the bottom-right panel of Figure 3 . The $J=2-1$ line of $\mathrm{C}_{4} \mathrm{H}^{-}$is detected in a single channel with a peak antenna temperature of $3.4 \mathrm{mK}$, which corresponds to $4.8 \sigma$ (where $\sigma$ is the rms noise of the baseline-subtracted spectrum). Using a Gaussian fit to this $\mathrm{C}_{4} \mathrm{H}^{-}$line with 1000 Monte Carlo noise replications, the central velocity was found to be $5.70 \pm 0.05 \mathrm{~km} \mathrm{~s}^{-1}$ with an FWHM of $0.43 \pm 0.13 \mathrm{~km} \mathrm{~s}^{-1}$. Within the errors, these parameters match those derived for $\mathrm{HC}_{3} \mathrm{~N}$ (given in Table 4), which adds confidence to our $\mathrm{C}_{4} \mathrm{H}^{-}$ detection. The integrated line intensity is $1.0 \pm 0.3 \mathrm{mK} \mathrm{km} \mathrm{s}^{-1}$, which corresponds to a column density of $N\left(\mathrm{C}_{4} \mathrm{H}^{-}\right)=$ $(8.0 \pm 2.4) \times 10^{9} \mathrm{~cm}^{-2}$ (assuming an excitation temperature of $10 \mathrm{~K}$ ). The neutral $\mathrm{C}_{4} \mathrm{H}$ line has an integrated intensity of

Table 3

Molecular Line Measurements and Anion-to-neutral Ratios

\begin{tabular}{lcccrr}
\hline \hline Source & $\begin{array}{c}\int T_{\mathrm{MB}} d v\left(\mathrm{C}_{6} \mathrm{H}^{-}\right)^{\mathrm{a}} \\
\left(\mathrm{mK} \mathrm{km} \mathrm{s}^{-1}\right)\end{array}$ & $\begin{array}{c}\int T_{\mathrm{MB}} d v\left(\mathrm{C}_{6} \mathrm{H}\right)^{\mathrm{b}} \\
\left(\mathrm{mK} \mathrm{km} \mathrm{s}^{-1}\right)\end{array}$ & $\begin{array}{c}N\left(\mathrm{C}_{6} \mathrm{H}^{-}\right) \\
\left(10^{10} \mathrm{~cm}^{-2}\right)\end{array}$ & $\begin{array}{c}N\left(\mathrm{C}_{6} \mathrm{H}\right) \\
\left(10^{11} \mathrm{~cm}^{-2}\right)\end{array}$ & $\begin{array}{c}\mathrm{C}_{6} \mathrm{H}^{-} / \mathrm{C}_{6} \mathrm{H} \\
(\%)\end{array}$ \\
\hline L1172 & $6.7(0.8)$ & $41.1(1.6)$ & $2.4(0.3)$ & $7.1(0.3)$ & $3.3(0.5)$ \\
L1251A & $6.5(1.0)$ & $43.6(2.1)$ & $2.3(0.4)$ & $7.6(0.4)$ & $3.0(0.6)$ \\
L1389 & $5.9(0.8)$ & $27.1(1.3)$ & $2.1(0.3)$ & $4.7(0.2)$ & $4.4(0.8)$ \\
L1495B & $9.6(1.0)$ & $141.6(2.2)$ & $3.4(0.4)$ & $24.6(0.4)$ & $1.4(0.2)$ \\
L1512 & $4.3(0.4)$ & $26.3(0.9)$ & $1.5(0.1)$ & $4.6(0.2)$ & $3.3(0.4)$ \\
TMC-1C & $13.6(1.1)$ & $88.1(1.7)$ & $4.8(0.4)$ & $15.3(0.3)$ & $3.1(0.3)$ \\
TMC-1 CP & $41.6(5.0)$ & $332.4(9.0)$ & $14.7(1.8)$ & $57.8(1.6)$ & $2.5(0.4)$ \\
\hline
\end{tabular}

Notes. Uncertainties given in parentheses are $\pm 68 \%$ Monte Carlo errors. Measurements for L1251A and L1512 supersede those of Cordiner et al. (2011) as explained in Section 4.

${ }^{\text {a }}$ Integrated intensities of average $\mathrm{C}_{6} \mathrm{H}^{-} J=10-9$ and $J=11-10$ spectra.

${ }^{\mathrm{b}}$ Integrated intensities summed over both hyperfine components of the $\mathrm{C}_{6} \mathrm{H} J=10.5-9.5, f$ transition. 

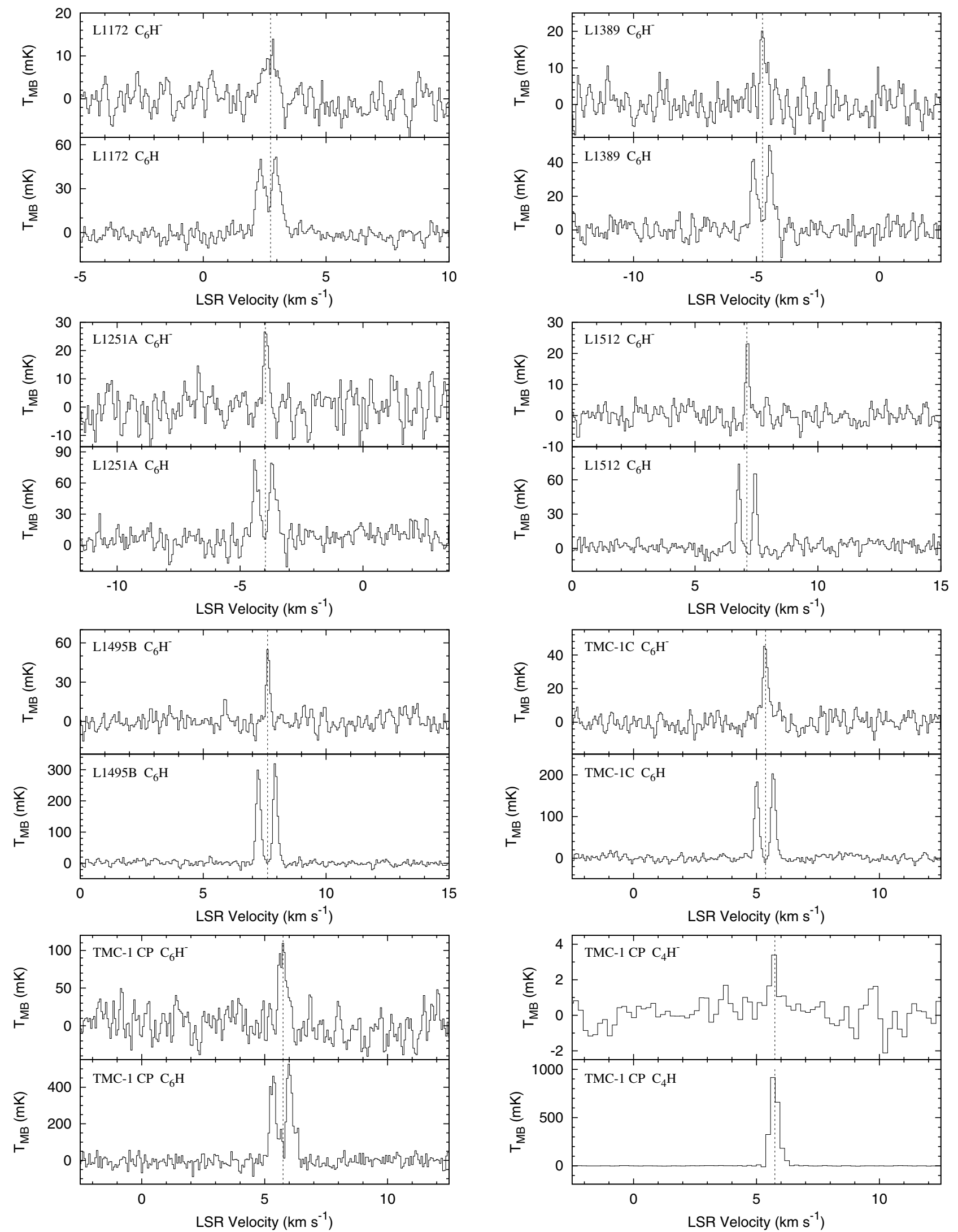

Figure 3. Observed $\mathrm{C}_{6} \mathrm{H}^{-}$spectra (average of $J=10-9$ and $J=11-10$ transitions), and $\mathrm{C}_{6} \mathrm{H} J=10.5-9.5, f$ spectra. The $\mathrm{C}_{6} \mathrm{H}$ velocity scale is given with respect to the (weighted) mean frequency of the two hyperfine components. For TMC-1, observed $\mathrm{C}_{4} \mathrm{H}^{-}(J=2-1)$ and $\mathrm{C}_{4} \mathrm{H}(N=2-1, J=1.5-0.5, F=2-1)$ spectra are also shown (bottom-right panel). Dashed vertical lines indicate the least-squares $\mathrm{HC}_{3} \mathrm{~N}$ velocities for each target. 
Table 4

$\mathrm{HC}_{3} \mathrm{~N}$ RADEX Fit Results

\begin{tabular}{lcccc}
\hline \hline Source & $\begin{array}{c}N\left(\mathrm{HC}_{3} \mathrm{~N}\right) \\
\left(10^{13} \mathrm{~cm}^{-2}\right)\end{array}$ & $\begin{array}{c}v\left(\mathrm{HC}_{3} \mathrm{~N}\right) \\
\left(\mathrm{km} \mathrm{s}^{-1}\right)\end{array}$ & $\begin{array}{c}\Delta v\left(\mathrm{HC}_{3} \mathrm{~N}\right) \\
\left(\mathrm{km} \mathrm{s}^{-1}\right)\end{array}$ & $\begin{array}{c}n_{\mathrm{H}_{2}} \\
\left(10^{4} \mathrm{~cm}^{-3}\right)\end{array}$ \\
\hline L1172 & 2.7 & 2.74 & 0.49 & $7.5(2.3)$ \\
L1251A & 3.3 & -3.97 & 0.31 & $2.1(0.4)$ \\
L1389 & 1.5 & -4.75 & 0.32 & $5.2(1.3)$ \\
L1495B & 8.2 & 7.62 & 0.19 & $1.1(0.3)$ \\
L1512 & 4.2 & 7.11 & 0.10 & $2.6(0.9)$ \\
TMC-1C & 6.4 & 5.37 & 0.18 & $1.1(0.4)$ \\
TMC-1 CP & 19.5 & 5.75 & 0.32 & $1.0(0.2)$ \\
\hline
\end{tabular}

Note. Uncertainties on $n_{\mathrm{H}_{2}}$ (in parentheses) were calculated assuming $\pm 1 \mathrm{~K}$ uncertainty on the kinetic temperature, but may be larger due to the possible effects of beam dilution.

$411.3 \pm 0.7 \mathrm{mK} \mathrm{km} \mathrm{s}^{-1}$, which requires $N\left(\mathrm{C}_{4} \mathrm{H}\right)=(6.91 \pm$ $0.01) \times 10^{14} \mathrm{~cm}^{-2}$. The corresponding anion-to-neutral ratio $\mathrm{C}_{4} \mathrm{H}^{-} / \mathrm{C}_{4} \mathrm{H}$ is $(1.2 \pm 0.4) \times 10^{-5}$. This value is consistent with the upper limit of $5.2 \times 10^{-5}$ measured by Agúndez et al. (2008), and our $\mathrm{C}_{4} \mathrm{H}$ column density also closely matches their value of $7.1 \times 10^{14} \mathrm{~cm}^{-2}$. Both $N\left(\mathrm{C}_{4} \mathrm{H}\right)$ and $N\left(\mathrm{C}_{4} \mathrm{H}^{-}\right)$are relatively insensitive to the adopted excitation temperature, and vary by only $12 \%$ over the range $5-10 \mathrm{~K}$.

\section{2. $\mathrm{HC}_{3} \mathrm{~N}$ Observations and Radiative Transfer Modeling}

Our $\mathrm{HC}_{3} \mathrm{~N}$ maps (Figures 1 and 2) show the presence of one or more compact molecular condensations in each source, and highlight a clumpy structure in this carbon-chain-rich gas. The peaks in $\mathrm{HC}_{3} \mathrm{~N}$ emission tend to coincide approximately with the locations of known prestellar cores and protostars, the positions of which are denoted with crosses and asterisks, respectively. As a result of the collapse and infall processes occurring in these objects, their densities are expected to be greater than the surrounding gas. This should result in increased collisional excitation of the $J=10$ level of $\mathrm{HC}_{3} \mathrm{~N}$, which has a critical density of $7 \times 10^{5} \mathrm{~cm}^{-3}$ (Buckle et al. 2006). Thus, the intensity variations shown in our $\mathrm{HC}_{3} \mathrm{~N}$ OSO maps represent a combination of variations in gas density and total $\mathrm{HC}_{3} \mathrm{~N}$ column.

Spectra of three rotational transitions of $\mathrm{HC}_{3} \mathrm{~N}$ for each source, observed at the locations targeted by our GBT anion survey, are shown in Figure 4. These span a range of upper-state energy levels $1.82-16.69 \mathrm{~cm}^{-1}(2.6-24.0 \mathrm{~K})$. In order to derive gas densities and velocities, the $\mathrm{HC}_{3} \mathrm{~N}$ spectra were subject to fitting using the RADEX radiative transfer code (van der Tak et al. 2007). Collisional transition rates were taken from the Leiden Atomic and Molecular Database ${ }^{6}$ (Schöier et al. 2005), which tabulates scaled versions of the original data of Green \& Chapman (1978). Hyperfine structure (HFS) was accounted for by assuming LTE population of the hyperfine levels in each $J$ state. The primary collision partner number density $\left(n_{\mathrm{H}_{2}}\right)$, molecular column density $(N)$, Doppler line FWHM $(\Delta v)$, and Doppler velocity $(v)$ were optimized for the spectra of each source using the MPFIT least-squares algorithm (Markwardt 2009). The best-fitting parameters are given in Table 4. The kinetic temperature was fixed at $10 \mathrm{~K}$, then varied by $\pm 1 \mathrm{~K}$ to produce the quoted error estimates on $n_{\mathrm{H}_{2}}$. For all sources, a temperature of $10 \mathrm{~K}$ produced a good fit to all five HFS components (the two weakest HFS components are not shown in Figure 4). The $\mathrm{HC}_{3} \mathrm{~N}$ Doppler velocities are shown with vertical dashed lines in Figure 3 and closely match the central velocities

\footnotetext{
6 http://www.strw.leidenuniv.nl/ moldata
}

of the detected hydrocarbons and anions. In several cases, the $\mathrm{HC}_{3} \mathrm{~N}$ line widths are very narrow; for L1512, $\Delta v=0.1 \mathrm{~km} \mathrm{~s}^{-1}$, which is only slightly greater than the $10 \mathrm{~K}$ thermal line width of $0.07 \mathrm{~km} \mathrm{~s}^{-1}$, indicating an unusual lack of turbulence, flows, or shears in the central $\sim 30^{\prime \prime}$ of this source.

The $\mathrm{C}_{6} \mathrm{H}^{-} / \mathrm{C}_{6} \mathrm{H}$ ratio is plotted as a function of $n_{\mathrm{H}_{2}}$ in Figure 5, and shows a positive correlation. There is considerable scatter and uncertainty on $n_{\mathrm{H}_{2}}$, but consistent with previous studies (Hirahara et al. 1992; Visser et al. 2002; Launhardt et al. 2010; Kirk et al. 2005, respectively), we find the lowest density in TMC-1, highest densities in L1172 and L1389, and an intermediate density in L1512. Our calculated $n_{\mathrm{H}_{2}}$ values for L1172, L1389, and L1512 are, however, systematically lower than previously derived values by up to two orders of magnitude. This discrepancy is likely indicative of a problem in our method, which can be attributed to the effects of different degrees of beam dilution affecting the different $\mathrm{HC}_{3} \mathrm{~N}$ line frequencies we observed. Given the larger $42^{\prime \prime}$ beam size for the $\mathrm{HC}_{3} \mathrm{~N}$ $J=10-9$ line observed with the OSO 20 m compared with the $27^{\prime \prime}$ GBT beam for the $J=3-2$ line, it is theoretically possible that the OSO data suffer up to an additional factor of 2.6 in beam dilution compared with that of the GBT, which could result in sufficient reduction of the derived $n_{\mathrm{H}_{2}}$ values to account for the observed discrepancies. Figures 1 and 2 (and Figure 7 of Pratap et al. 1997) show that the $\mathrm{HC}_{3} \mathrm{~N}$ emission from all our observed sources is distributed over an area larger than the OSO beam, which suggests that beam dilution may not be so severe. However, given the lack of spatial information on the extent of the $J=10-9$ emission on size scales less than the OSO beam size, it is impossible to quantify the importance of beam dilution in our RADEX calculations, so the $n_{\mathrm{H}_{2}}$ values given in Table 4 should be treated with caution. More accurate estimates for $n_{\mathrm{H}_{2}}$ could be obtained from $\mathrm{HC}_{3} \mathrm{~N} J=10-9$ observations using a telescope with a smaller beam (such as the IRAM $30 \mathrm{~m}$ ), which would be a better match to that of our GBT observations.

\section{DISCUSSION}

\section{1. $\mathrm{C}_{6} \mathrm{H}^{-}$}

Since the 2006 discovery of $\mathrm{C}_{6} \mathrm{H}^{-}$in TMC-1, there have been seven reported detections of this anion in various parts of the Taurus-Auriga molecular cloud complex (including our latest detections in L1495B, L1512, and TMC-1C). Thus far, there have been only two detections of interstellar anions outside of this small region (Sakai et al. 2010; Cordiner et al. 2011), which highlights the importance of our new discoveries of $\mathrm{C}_{6} \mathrm{H}^{-}$in the vicinity of L1172 SMM (a prestellar core in Cepheus) and L1389 SMM1 (a prestellar/protostellar core inside Bok Globule CB17 in Camelopardalis). These new detections confirm that anions are indeed widespread in star-forming regions outside of Taurus, and that $\mathrm{C}_{6} \mathrm{H}^{-}$appears to be ubiquitous in dense interstellar clouds wherever its parent neutral is present.

Myers et al. (1988) detected an outflow in L1172 that Visser et al. (2002) identified as originating from a protostar (L1172 SMM1) that lies about $30^{\prime \prime}$ south of our targeted position. They also detected a dense core (presumed prestellar), about $50^{\prime \prime}$ southeast of our target, with $N\left(\mathrm{H}_{2}\right)=6 \times 10^{21} \mathrm{~cm}^{-2}$. If this column density also applies to our observed position, the $\mathrm{C}_{6} \mathrm{H}^{-}$ abundance is $4 \times 10^{-12}$, which is a factor of a few less than in TMC-1, but an order of magnitude greater than in L1512, L1521F, and L1544 (see Cordiner et al. 2012, and references therein), suggesting a relatively high absolute anion abundance in L1172. 


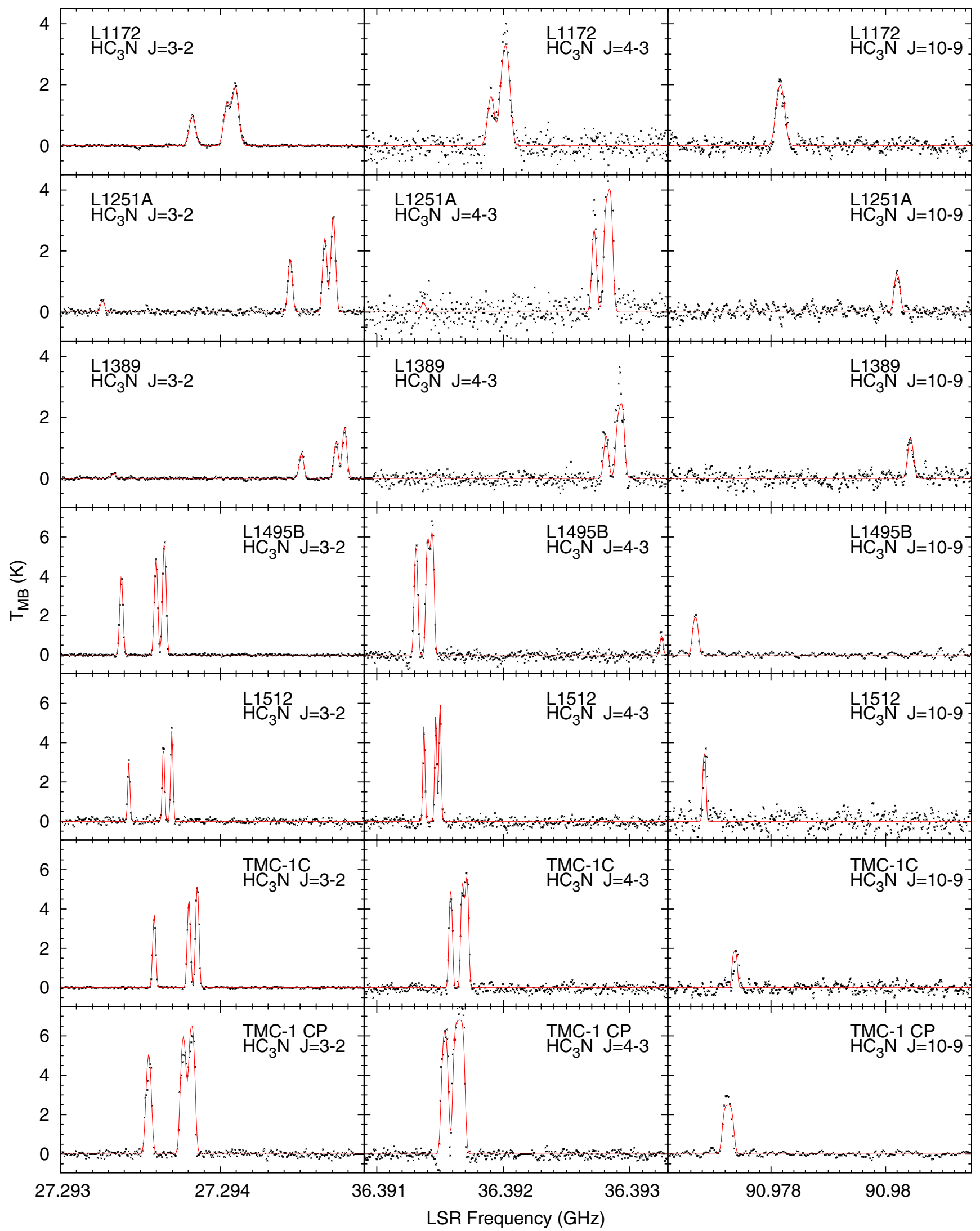

Figure 4. Observed $\mathrm{HC}_{3} \mathrm{~N}$ spectra (black points) with RADEX least-squares models overlaid (red curves). Frequency-switching residuals have been masked from the observed data. Three main hyperfine peaks are visible in the $J=3-2$ and $J=4-3$ spectra. Model parameters are given in Table 4.

(A color version of this figure is available in the online journal.) 


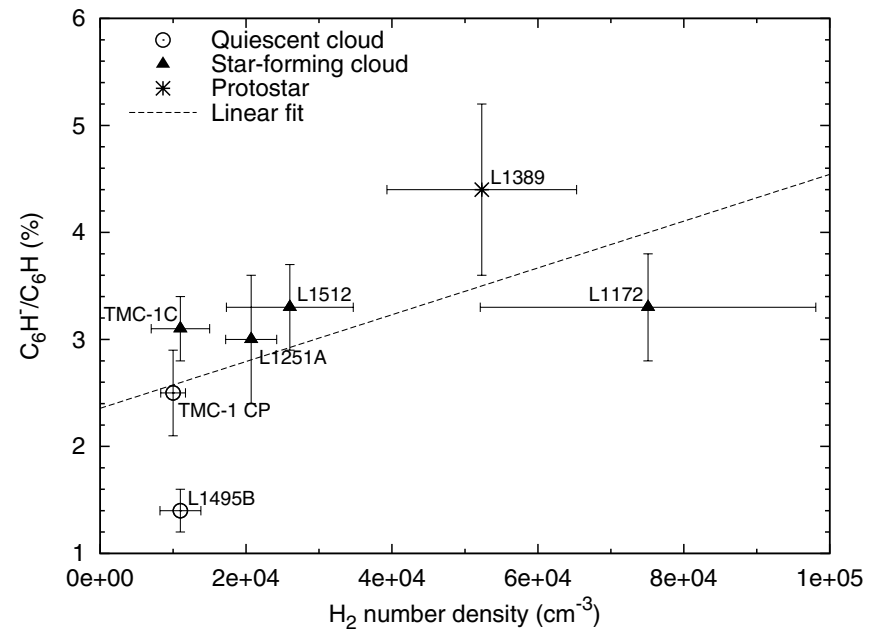

Figure 5. $\mathrm{C}_{6} \mathrm{H}^{-}$anion-to-neutral ratio vs. $\mathrm{H}_{2}$ number density $\left(n_{\mathrm{H}_{2}}\right)$. The dashed line shows linear least-squares fit.

By utilizing the available physical and chemical information on the interstellar clouds for which $\mathrm{C}_{6} \mathrm{H}^{-}$has so far been detected (as summarized for our observed targets in Section 2), it is possible to examine the $\mathrm{C}_{6} \mathrm{H}^{-} / \mathrm{C}_{6} \mathrm{H}$ ratio with respect to each cloud's chemical and dynamical evolutionary state. The evolutionary states of the observed clouds are given in Table 1, and are shown by the different symbols on the plot in Figure 5. There is a clear division in the anion-to-neutral ratio between young, "quiescent" and older, "star-forming" cores: for the quiescent, chemically young cores L1495B and TMC-1 (CP), $\mathrm{C}_{6} \mathrm{H}^{-} / \mathrm{C}_{6} \mathrm{H}<3 \%$, and for the star-forming cores/protostars, $\mathrm{C}_{6} \mathrm{H}^{-} / \mathrm{C}_{6} \mathrm{H} \geqslant 3 \%$. L1495B is the most chemically young and quiescent of our sources (Hirota et al. 2004), with no evidence for nearby star formation, and has the lowest $\mathrm{C}_{6} \mathrm{H}^{-} / \mathrm{C}_{6} \mathrm{H}$ ratio in our sample $(1.4 \% \pm 0.2 \%)$. The very low luminosity, low mass protostar (VeLLO) L1389 SMM1, on the other hand, evidently contains among the most evolved matter in our sample (having passed through the quiescent and star-forming stages), and has the highest anion-to-neutral ratio $\left(\mathrm{C}_{6} \mathrm{H}^{-} / \mathrm{C}_{6} \mathrm{H}=4.4 \% \pm 0.8 \%\right)$. This value is similar to the value of $4.0 \% \pm 1.0 \%$ found in L1521F by Gupta et al. (2009), which also contains a VeLLO (Bourke et al. 2006). Comparing our results with other sources from the literature, L1527 (Sakai et al. 2007) contains a somewhat more-evolved Class $0 / \mathrm{I}$ protostar, and this source has a significantly larger $\mathrm{C}_{6} \mathrm{H}^{-} / \mathrm{C}_{6} \mathrm{H}$ ratio of $9.3 \% \pm 2.9 \%$. Thus, we confirm the apparent relationship identified by Cordiner et al. (2011) between the evolutionary state of interstellar matter and its $\mathrm{C}_{6} \mathrm{H}^{-}$anion-to-neutral ratio. The quiescent cloud Lupus-1A observed by Sakai et al. (2010) also fits the trend (with $\mathrm{C}_{6} \mathrm{H}^{-} / \mathrm{C}_{6} \mathrm{H}=2.1 \% \pm 0.6 \%$ ), as does the very low luminosity protostar Chamaeleon MMS1 (with its upper limit of $\mathrm{C}_{6} \mathrm{H}^{-} / \mathrm{C}_{6} \mathrm{H}<10 \%$; Cordiner \& Charnley 2012). The dense prestellar core L1544 may deviate, however, with a relatively low ratio of $2.5 \% \pm 0.8 \%$ (Gupta et al. 2009).

The observed $\mathrm{C}_{6} \mathrm{H}^{-} / \mathrm{C}_{6} \mathrm{H}$ trend and its correlation with $n_{\mathrm{H}_{2}}$ shown in Figure 5 can be understood in the context of the theoretical study of Cordiner et al. (2012), who identified the effects of depletion on the $\mathrm{C}_{6} \mathrm{H}^{-}$anion-to-neutral ratio, the degree of which is related to both the density and chemical age of the cloud. Depletion occurs as atoms and molecules collide with and stick to dust grains, and thus proceeds faster in denser media. Increased depletion affects the anion-toneutral ratio in two ways: (1) the free electron abundance goes up so that more $\mathrm{C}_{6} \mathrm{H}^{-}$is produced by radiative electron attachment and (2) the atomic $\mathrm{O}$ and $\mathrm{H}$ abundances go down, thus reducing the anion destruction rate. Our $\mathrm{C}_{6} \mathrm{H}^{-}$observations agree with this theory, particularly for the quiescent cores and the protostars, which are believed to lie at opposite ends of the interstellar chemical/dynamic evolutionary path. Measured anion-to-neutral ratios, however, are not sufficiently accurate to draw a clear distinction among the (moderately evolved) prestellar cores and star-forming gas clouds of L1172, L1251A, L1512, and TMC-1C. These clouds likely contain gas that spans a range of densities and degrees of chemical evolution. More accurate measurements of density, depletion, and the $\mathrm{C}_{6} \mathrm{H}^{-} / \mathrm{C}_{6} \mathrm{H}$ ratio at high spatial resolution will be required in order to confirm the utility of the anion-to-neutral ratio as a measure of the evolutionary state of interstellar clouds.

\section{2. $\mathrm{C}_{4} \mathrm{H}^{-}$}

Our detection of $\mathrm{C}_{4} \mathrm{H}^{-}$constitutes the smallest reported column density for this molecule in any source to date. Consistent with the trend described above for $\mathrm{C}_{6} \mathrm{H}^{-}$, the $\mathrm{C}_{4} \mathrm{H}^{-}$anion-toneutral ratio of $(1.2 \pm 0.4) \times 10^{-5}$ in TMC-1 (CP) is about nine times smaller than observed in L1527 by Agúndez et al. (2008). Theory regarding $\mathrm{C}_{4} \mathrm{H}^{-}$chemistry is less well understood than for $\mathrm{C}_{6} \mathrm{H}^{-}$, but it seems plausible to again ascribe the lower value in TMC-1 (and the moderately low value of $(8.8 \pm 5.3) \times 10^{-5}$ in Lupus-1A; Sakai et al. 2010), to chemical effects resulting from the lower densities and younger evolutionary states of these sources compared with L1527.

The very low observed $\mathrm{C}_{4} \mathrm{H}^{-}$anion-to-neutral ratios compared with $\mathrm{C}_{6} \mathrm{H}^{-}$are at variance with chemical models that consider the formation of $\mathrm{C}_{4} \mathrm{H}^{-}$to be by radiative electron attachment to $\mathrm{C}_{4} \mathrm{H}$ at the rate calculated by Herbst \& Osamura (2008). Recent ab initio calculations by V. Kokoouline et al. (2012, private communication) show that the $\mathrm{C}_{4} \mathrm{H}$ radiative attachment rate may be several orders of magnitude less than previously thought, and based on observations of the $\mathrm{C}_{4} \mathrm{H}^{-} / \mathrm{C}_{4} \mathrm{H}$ ratio in L1527, Agúndez et al. (2008) calculated a $\mathrm{C}_{4} \mathrm{H}$ radiative attachment rate a factor of 122 less than the theoretical value of Herbst \& Osamura (2008). However, using the laboratory data of Eichelberger et al. (2007), Cordiner et al. (2012) identified that a significant pathway to smaller hydrocarbon anions is via reactions of larger anions with atomic oxygen. Thus, an important source of $\mathrm{C}_{4} \mathrm{H}^{-}$is the reaction

$$
\mathrm{C}_{5} \mathrm{H}^{-}+\mathrm{O} \longrightarrow \mathrm{C}_{4} \mathrm{H}^{-}+\mathrm{CO} \text {. }
$$

As a consequence of this (and the analogous reaction that forms $\mathrm{C}_{5} \mathrm{H}^{-}$from $\mathrm{C}_{6} \mathrm{H}^{-}+\mathrm{O}$ ), using the model of Cordiner et al. (2012) at $n_{\mathrm{H}_{2}}=10^{4} \mathrm{~cm}^{-3}$ (applicable to TMC-1), it is possible to set the radiative attachment rates for both $\mathrm{C}_{4} \mathrm{H}$ and $\mathrm{C}_{5} \mathrm{H}$ to zero and still produce an absolute $\mathrm{C}_{4} \mathrm{H}^{-}$abundance and $\mathrm{C}_{4} \mathrm{H}^{-} / \mathrm{C}_{4} \mathrm{H}$ ratio that are within a factor of a few of the observed values. It is therefore plausible that radiative electron attachment is not the dominant route to the formation of $\mathrm{C}_{4} \mathrm{H}^{-}$in molecular clouds.

\section{CONCLUSION}

We conducted a search for the carbon-chain anion $\mathrm{C}_{6} \mathrm{H}^{-}$in seven nearby molecular clouds selected based on their strong $\mathrm{HC}_{3} \mathrm{~N}$ emission. $\mathrm{C}_{6} \mathrm{H}^{-}$and $\mathrm{C}_{6} \mathrm{H}$ were detected in all sources, with a mean anion-to-neutral ratio of $3.0 \%$ (standard deviation $0.92 \%$ ). Combined with the four previous $\mathrm{C}_{6} \mathrm{H}^{-}$detections in 
other sources, we deduce that anions are ubiquitous in the ISM wherever sufficient $\mathrm{C}_{6} \mathrm{H}$ is present to be easily detectable.

From the combined sample of 11 known interstellar sources with $\mathrm{C}_{6} \mathrm{H}^{-}$detections, we confirm the trend identified by Cordiner et al. (2011) for anion-to-neutral ratios to be smaller in younger, less dense environments and larger in older, denser environments. This is consistent with the theory of Cordiner et al. (2012) that anion-to-neutral ratios are sensitive to the depletion of atomic $\mathrm{O}$ and $\mathrm{H}$, and to the electron density, both of which become larger in denser, more chemically evolved cores. Molecular hydrogen number densities were derived for each source based on radiative transfer fits to observed $\mathrm{HC}_{3} \mathrm{~N}$ spectra and a positive correlation was found between $\mathrm{C}_{6} \mathrm{H}^{-} / \mathrm{C}_{6} \mathrm{H}$ and $n_{\mathrm{H}_{2}}$.

We report the first detection of $\mathrm{C}_{4} \mathrm{H}^{-}$in TMC-1, and derive an anion-to-neutral ratio $\mathrm{C}_{4} \mathrm{H}^{-} / \mathrm{C}_{4} \mathrm{H}=(1.2 \pm 0.4) \times 10^{-5}(=$ $0.0012 \% \pm 0.0004 \%)$. This is the smallest anion-to-neutral ratio yet observed and confirms a problem with the theoretical value for the $\mathrm{C}_{4} \mathrm{H}$ electron attachment rate, which is apparently several orders of magnitude too large. The observed $\mathrm{C}_{4} \mathrm{H}^{-}$may instead be formed by reactions of the larger hydrocarbon anions $\left(\mathrm{C}_{6} \mathrm{H}^{-}\right.$ and $\mathrm{C}_{5} \mathrm{H}^{-}$) with atomic oxygen.

Given the widespread presence of anions in space, a combination of further dedicated laboratory, observational, and theoretical studies will be required in order to better understand their behavior and thus their full importance in astrochemistry. Measurements of radiative electron attachment rates for hydrocarbons and branching ratios for reactions of anions with atomic oxygen will be of particular importance in this regard.

This research was supported by the NASA Exobiology Program and the NASA Astrobiology Institute through the Goddard Center for Astrobiology. We gratefully acknowledge the assistance of Tom Millar and Catherine Walsh during the 2010 Onsala $\mathrm{HC}_{3} \mathrm{~N}$ observing run. J.V.B. thanks John Richer for support. The National Radio Astronomy Observatory is a facility of the National Science Foundation operated under cooperative agreement by Associated Universities, Inc.

\section{REFERENCES}

Agúndez, M., Fonfría, J. P., Cernicharo, J., Pardo, J. R., \& Guélin, M. 2008, A\&A, 479, 493

Bell, M. B., Feldman, P. A., Watson, J. K. G., et al. 1999, ApJ, 518, 740

Bourke, T. L., Myers, P. C., Evans, N. J., et al. 2006, ApJL, 649, L37

Brünken, S., Gupta, H., Gottlieb, C. A., McCarthy, M. C., \& Thaddeus, P. 2007, ApJL, 664, L43

Buckle, J. V., Rodgers, S. D., Wirström, E. S., et al. 2006, FaDi, 133, 63

Chen, X., Arce, H., Dunham, M., et al. 2012, ApJ, 751, 89

Clemens, D. P., \& Barvainis, D. P. 1988, ApJS, 68, 257

Cordiner, M. A., \& Charnley, S. B. 2012, ApJ, 749, 120
Cordiner, M. A., Charnley, S. B., Buckle, J. V., Walsh, C., \& Millar, T. J. 2011, ApJL, 730, L18

Cordiner, M. A., Charnley, S. B., Wirström, E. S., \& Smith, R. G. 2012, ApJ, 744,131

Cordiner, M. A., Millar, T. J., Walsh, C., et al. 2008, in IAU Symp. 251, Organic Matter in Space, ed. S. Kwok \& S. Stanford (Cambridge: Cambridge Univ. Press), 157

Dalgarno, A., \& McCray, R. A. 1973, ApJ, 181, 95

de Zafra, R. L. 1971, ApJ, 170, 165

Di Francesco, J., Johnstone, D., Kirk, H., MacKenzie, T., \& Ledwosinska, E. 2008, ApJS, 175, 227

Eichelberger, B., Snow, T. P., Barckholtz, C., \& Bierbaum, V. M. 2007, ApJ, 667,1283

Elias, J. H. 1978, ApJ, 224, 857

Federman, S. R., Huntress, W. T., Jr., \& Prasad, S. S. 1990, ApJ, 354, 504

Gottlieb, C. A., Gottlieb, E. W., Thaddeus, P., \& Kawamura, H. 1983, ApJ, 275,916

Green, S., \& Chapman, S. 1978, ApJS, 37, 169

Gupta, H., Brünken, S., Tamassia, F., et al. 2007, ApJL, 655, L57

Gupta, H., Gottlieb, C. A., McCarthy, M. C., \& Thaddeus, P. 2009, ApJ, 691,1494

Harada, N., \& Herbst, E. 2008, ApJ, 685, 272

Herbst, E. 1981, Natur, 289, 656

Herbst, E., \& Osamura, Y. 2008, ApJ, 679, 1670

Hirahara, Y., Suzuki, H., \& Yamamoto, S. 1992, ApJ, 394, 539

Hirota, T., Hiroyuki, M., \& Yamamoto, S. 2004, ApJ, 617, 399

Jørgensen, J. K., Schöier, F. L., \& van Dishoeck, E. F. 2002, A\&A, 389, 908

Kirk, J. M., Ward-Thompson, D., \& André, P. 2005, MNRAS, 360, 1506

Kun, M., \& Prusti, T. 1993, A\&A, 272, 235

Launhardt, R., Nutter, D., Ward-Thompson, D., et al. 2010, ApJS, 188, 139

Lee, J., Lee, H., Shinn, J., et al. 2010, ApJ, 709, 74

Lis, D. C., Roueff, E., Gerin, M., et al. 2002, ApJL, 571, L55

Markwardt, C. B. 2009, in ASP Conf. Ser. 411, Astronomical Data Analysis Software and Systems XVIII, Quebec, Canada, ed. D. Bohlender, P. Dowler, \& D. Durand (San Francisco, CA: ASP), 251

McCarthy, M. C., Gottlieb, C. A., Gupta, H. C., \& Thaddeus, P. 2006, ApJL, 652, L141

McCarthy, M. C., \& Thaddeus, P. 2005, JChPh, 122, 174308

McCarthy, M. C., \& Thaddeus, P. 2008, JChPh, 129, 054314

Millar, T. J., \& Herbst, E. 1994, A\&A, 288, 561

Millar, T. J., Walsh, C., Cordiner, M. A., Ní Chuimín, R., \& Herbst, E. 2007, ApJL, 662, L87

Müller, H. S. P., Schlöder, F., Stutzki, J., \& Winnewisser, G. 2005, JMoSt, 742, 215

Myers, P. C., Heyer, M., Snell, R., \& Goldsmith, P. 1988, ApJ, 324, 907

Pavlyuchenkov, Y., Wiebe, D., Launhardt, R., \& Henning, T. 2006, ApJ, 645, 1212

Pratap, P., Dickens, J. E., Snell, R. L., et al. 1997, ApJ, 486, 862

Remijan, A. J., Hollis, J. M., Lovas, F. J., et al. 2007, ApJL, 664, L47

Sakai, N., Sakai, T., Osamura, Y., \& Yamamoto, S. 2007, ApJL, 667, L71

Sakai, N., Shiino, T., Hirota, T., Sakai, T., \& Yamamoto, S. 2010, ApJL, 718, L49

Sarre, P. J. 1980, JCP, 77, 769

Schöier, F. L., van der Tak, F. F. S., van Dishoeck, E. F., \& Black, J. H. 2005, A\&A, 432, 369

Thorwirth, S., Müller, H. S. P., \& Winnewisser, G. 2000, JMoSp, 204, 133

van der Tak, F. F. S., Black, J. H., Schöier, F. L., Jansen, D. J., \& van Dishoeck, E. F. 2007 , A\&A, 468, 627

Visser, A. E., Richer, J. S., \& Chandler, C. J. 2002, ApJ, 124, 2756

Ward-Thompson, D., Scott, P. F., Hills, R. E., \& André, P. 1994, MNRAS, 268, 276

Woodin, R., Foster, M. S., \& Beauchamp, J. L. 1980, JChPh, 72, 4223 